\title{
Résumé sur l'utilisation et l'impact de la recherche: Traduire les données probantes en impact: améliorer la santé en Afrique de l'Ouest francophone et dans le Sahel
}

Population Council

Follow this and additional works at: https://knowledgecommons.popcouncil.org/

series_newsletters_researchimpact

How does access to this work benefit you? Let us know!

\section{Recommended Citation}

"Résumé sur l'utilisation et l'impact de la recherche: Traduire les données probantes en impact: améliorer la santé en Afrique de l'Ouest francophone et dans le Sahel," Research Utilization and Impact Brief. Washington, DC: Population Council, 2022. 


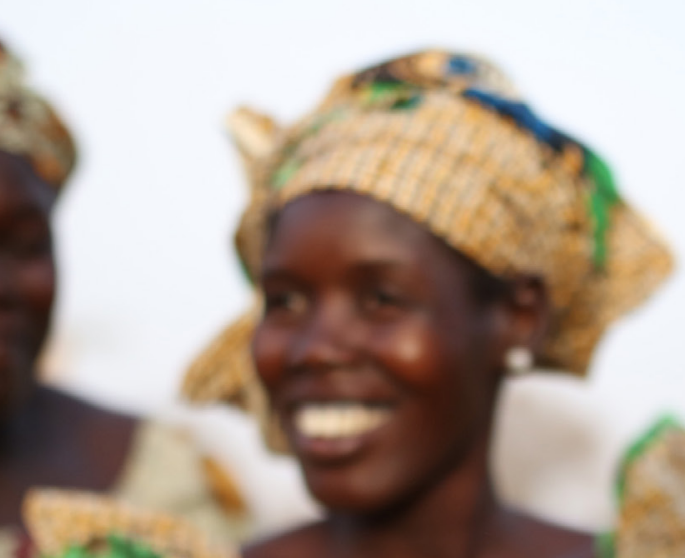

\section{RÉSUMÉ SUR L'UTILISATION ET L'IMPACT DE LA RECHERCHE}

\section{Traduire les données probantes en impact: améliorer la santé en Afrique de l'Ouest francophone et dans le Sahel}

\section{Résumé}

- Au cours des trois dernières décennies, le Population Council a œuvré pour faire avancer les efforts déployés à l'échelle infranationale, nationale et régionale afin d'améliorer la santé et promouvoir des politiques fondées sur des données probantes dans neuf pays d'Afrique de l'Ouest francophone.

- Le Population Council a joué un rôle majeur dans le renforcement des compétences techniques du personnel local et dans l'appui accordé aux partenariats régionaux de longue date, notamment l'Unité de Coordination du Partenariat de Ouagadougou et l'Organisation Ouest Africaine de la Santé.

- Cet engagement soutenu a contribué à générer des évidences, à renforcer les capacités institutionnelles et individuelles et la collaboration régionale pour renforcer les politiques et les programmes en vue d'élargir l'accès à la contraception, lutter contre les mutilations génitales féminines et l'excision (MGF/E), améliorer les opportunités économiques pour les filles et créer un environnement favorable aux soins de santé reproductive complets.
L'Afrique de l'Ouest francophone est une région dynamique et diversifiée qui compte environ 80 millions d'habitants. Forts de leurs identités nationales et de la collaboration régionale, les pays d'Afrique de l'Ouest francophone ont travaillé ensemble pour poursuivre des objectifs communs en matière de santé et de développement tels que l'amélioration de l'accès à la planification familiale/à la santé reproductive (PF/SR); la satisfaction des besoins en matière de santé, d'éducation et d'emploi d'une importante population de jeunes; et pour adresser des défis tels que le changement climatique et les conflits. Cette région intégrée sur le plan politique et linguistique est caractérisée par des partenariats économiques et des pactes de coopération, avec des accords réglementaires régionaux permettant de renforcer le partage et l'adoption d'outils, d'approches et de meilleures pratiques. Bien que la région ait historiquement bénéficié d'un appui moindre de la part des bailleurs de fonds en comparaison avec d'autres pays du Sud, des partenaires comme le Population Council ont maintenu leur engagement à cultiver des partenariats durables, en renforçant les capacités locales et en générant et en utilisant des données probantes pour aborder des enjeux importants. 
Le Population Council a commencé à s'engager en Afrique de l'Ouest francophone dans les années 1980, en réponse aux demandes des pays en matière de renforcement des capacités en PF/ SR et dans d'autres domaines de la santé. Les modèles de longue collaboration avec les gouvernements nationaux, qui en ont résulté pendant des décennies, y compris leurs ministères de la Santé (MS) et autres (par exemple, de la Famille, de la Jeunesse), les autorités locales, les organisations non gouvernementales nationales et internationales (ONG), les bailleurs de fonds et les agences normatives continuent de renforcer les partenariats vitaux des pays hôtes. Durant cette période, le Population Council a également entretenu des collaborations avec des entités régionales telles que l'Organisation ouest-africaine de la santé (OOAS), et l' Unité de Coordination du Partenariat de Ouagadougou (UCPO), le bras opérationnel du PO établi en 2012. Le Population Council a pris part à de nombreuses activités menées par la Coalition pour les Produits de Santé de la Reproduction (RHSC) en Afrique de l'Ouest francophone et il a apporté sa contribution à son Forum pour la Sécurité Contraceptive en Afrique Francophone (SECONAF) en diffusant les résultats des recherches conduites par ses membres.
Le travail du Population Council en Afrique de l'Ouest francophone et dans le Sahel repose sur des recherches et des collaborations dans 11 pays: Bénin, Burkina Faso, Cameroun, Tchad, Côte d'Ivoire, Guinée, Mali, Mauritanie, Niger, Sénégal et Togo. Dans ces contextes, l'expertise, les directives des pays hôtes et les partenariats font partie intégrante du travail du Population Council, ce qui favorise la collaboration et les investissements à tous les niveaux des systèmes de santé et crée les conditions idoines pour une action durable et menée au niveau local. Parmi les exemples de sujets de recherche figurent la qualité des soins au sein des services de santé clinique et les services de santé reproductive des jeunes; les campagnes dans les média de masse et sur les réseaux sociaux en faveur du changement social et comportemental (CSC) ; les programmes d'autonomisation des adolescentes ; l'optimisation des ressources humaines pour la santé par le transfert/partage des tâches ; ainsi que la prévention des pratiques néfastes comme le mariage des enfants, les mutilations génitales féminines et l'excision (MGF/E).
Le Population Council fournit un soutien stratégique et technique aux partenaires en concevant et en mettant en œuvre des recherches pertinentes et innovantes et en favorisant l'utilisation de données probantes pour améliorer les politiques et les programmes. Population Council aborde des questions sensibles qui affectent la santé et les droits des femmes, des hommes et des enfants. Grâce au travail de Population Council, le Sénégal et d'autres pays de l'Afrique de l'ouest francophone ont mis en œuvre des politiques fondées sur des données probantes et des programmes pour aborder la santé des populations vulnérables, prévenir les MGF/E, répondre aux besoins des adolescents en matière de santé de la reproduction et répondre aux besoins non satisfaits en matière de PF et de grossesses non désirées (y compris les soins postavortement et l'avortement sans risque).

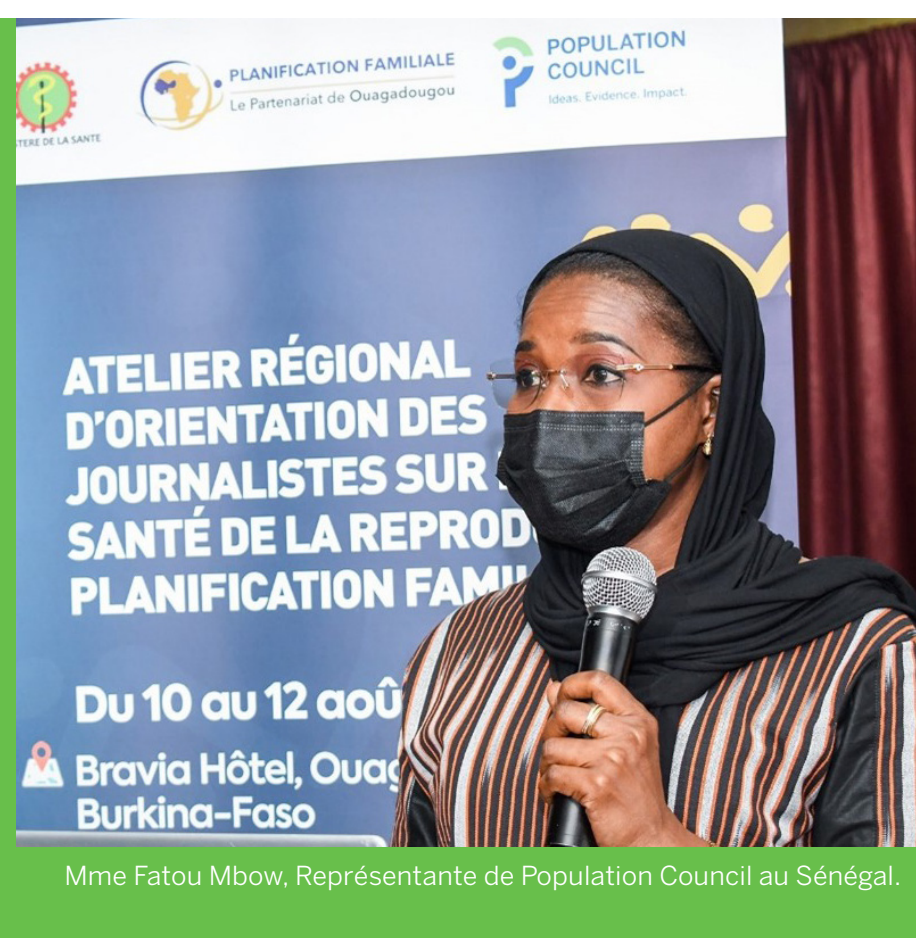




\section{PROJETS ACTUELS ET RÉCENTS DU POPULATION COUNCIL EN AFRIQUE DE L'OUEST FRANCOPHONE}

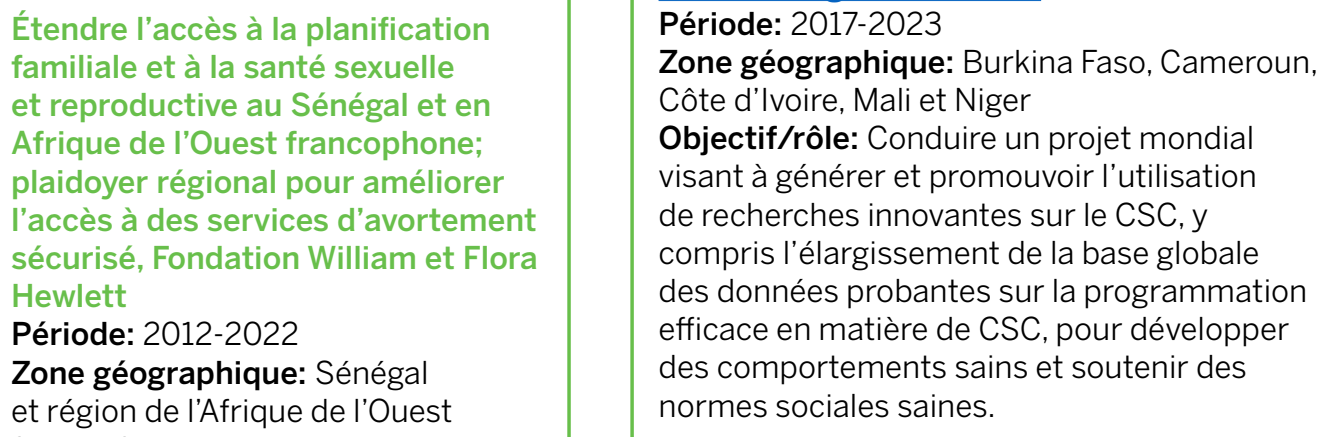

Étendre l'accès à la planification familiale et à la santé sexuelle et reproductive au Sénégal et en Afrique de l'Ouest francophone; plaidoyer régional pour améliorer l'accès à des services d'avortement sécurisé, Fondation William et Flora Hewlett

Période: 2012-2022

Zone géographique: Sénégal et région de l'Afrique de l'Ouest

Période: 2017-2023

Zone géographique: Burkina Faso, Cameroun, Côte d'Ivoire, Mali et Niger

Objectif/rôle: Conduire un projet mondial visant à générer et promouvoir l'utilisation de recherches innovantes sur le CSC, y compris l'élargissement de la base globale des données probantes sur la programmation efficace en matière de CSC, pour développer des comportements sains et soutenir des normes sociales saines. francophone

Objectif/rôle: Générer des évidences et fournir une assistance technique, y compris une collaboration avec le PO et d'autres partenaires clés et appui au Forum de la CEDEAO des Bonnes Pratiques en Santé, co-organisé par la Communauté économique des États de l'Afrique de l'Ouest (CEDEAO) et I'OOAS.

\section{Breakthrough RESEARCH, USAID}

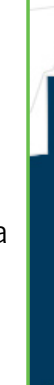

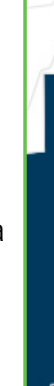
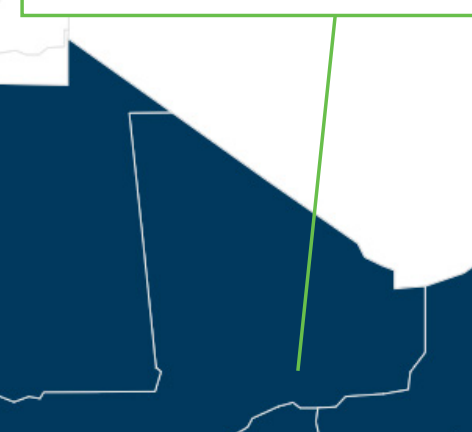

\author{
Projet pour \\ l'autonomisation des \\ femmes et le dividende \\ démographique au Sahel \\ (SWEDD), UNFPA-West and \\ Central Africa Regional \\ Office
}

Période: 2019-2024

Zone géographique: Bénin, Burkina Faso, Cameroun, Tchad, Côte d'Ivoire, Guinée, Mali, Mauritanie et Niger Objectif/rôle: Fournir une assistance technique et appui à la mise en œuvre d'un projet régional consacré aux activités axées sur les adolescentes.

\section{AmplifyPF, USAID}

Période: 2018-2023

Zone géographique: Burkina Faso, Côte d'Ivoire, Niger et Togo

\section{Objectif/rôle: Sous-}

bénéficiaire du projet de Pathfinder International (bénéficiaire principal) sur l'assurance qualité et le suivi pour élargir l'accès et le recours aux services de planification familiale de qualité en Afrique de l'Ouest.
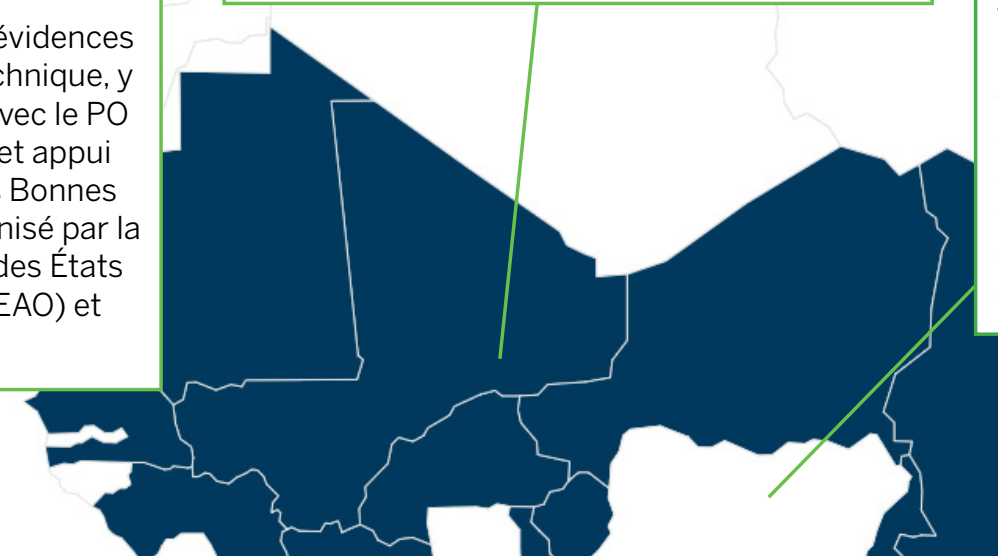

ré




\section{CHRONOLOGIE: HISTORIQUE DU PARCOURS DU POPULATION COUNCIL EN AFRIQUE DE L'OUEST FRANCOPHONE}
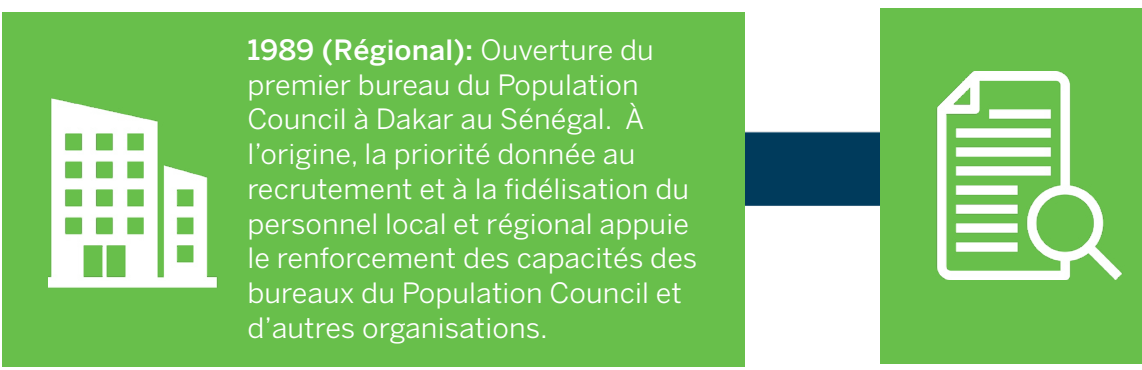

1994 (Sénégal): A mis en

place un partenariat avec le MS

du Sénégal pour conduire la première analyse situationnelle nationale sur les points de prestation de services de SR. Le Population Council a appuyé la conduite d'une deuxième analyse situationnelle en 1998.

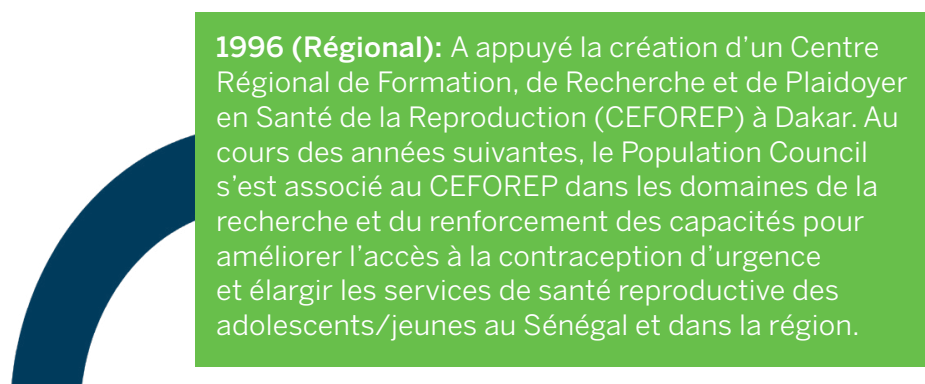

Fin des années 1990 (Burkina Faso): A répliqué l'intervention de dialogue et d'engagement communautaire de Tostan pour éliminer les MGF/E au Burkina Faso, en testant sa faisabilité et son efficacité. Une autre ONG burkinabé, Mwangaza Action, a par la suite adapté et mis en œuvre ce modèle dans 23 villages.

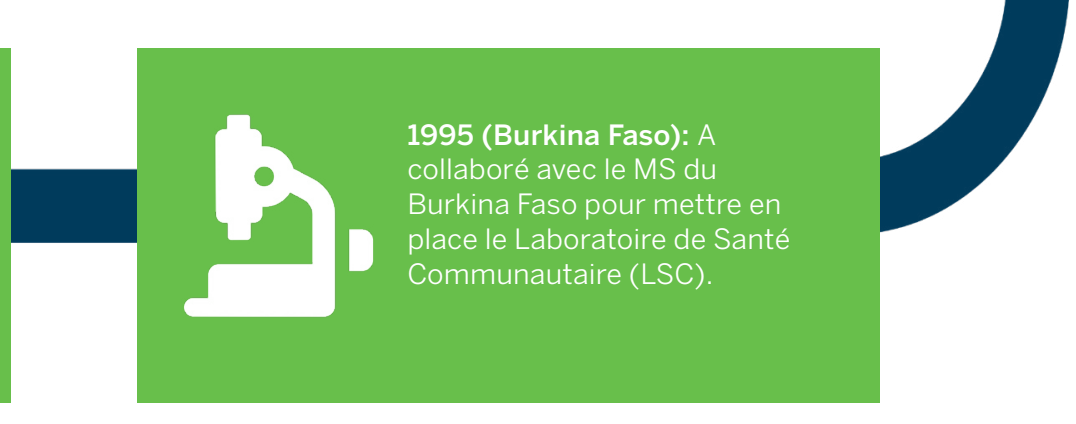

1997-2008 (Global): A dirigé le programme de recherches opérationnelles sur le VIH/sida, Horizons, financé par I'USAID, avec des activités menées au Sénégal ciblant les hommes qui ont des rapports sexuels avec des hommes. L'accent mis très tôt sur la génération d'un débat public sur les besoins de cette population marginalisée a renforcé les efforts du Population Council pour légitimer et aborder des thèmes sensibles comme les MGF/E et les soins après avortement.

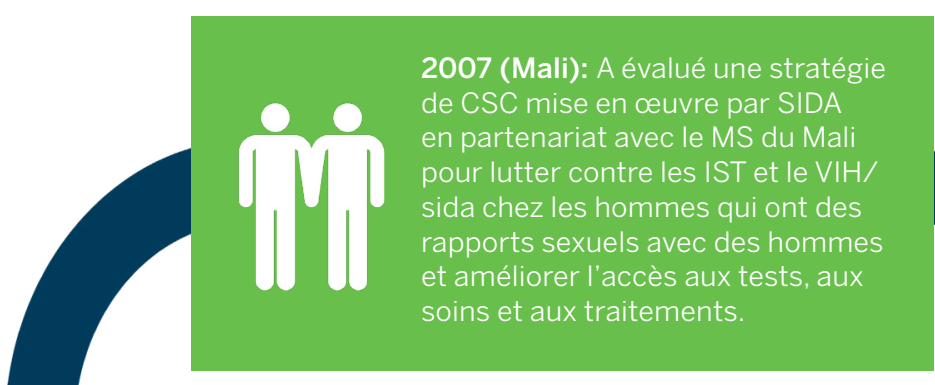

2005-2008 (Régional): Sur la base d’un travail antérieur consacré à un plan stratégique pour l'utilisation du préservatif féminin au Sénégal, a établi un partenariat avec les gouvernements nationaux et les bureaux de pays du FNUAP pour identifier les stratégies adaptées en vue de l'intégration des préservatifs féminins et masculins dans les programmes nationaux de SR et de VIH/ sida au Bénin, au Burkina Faso et en Mauritanie.

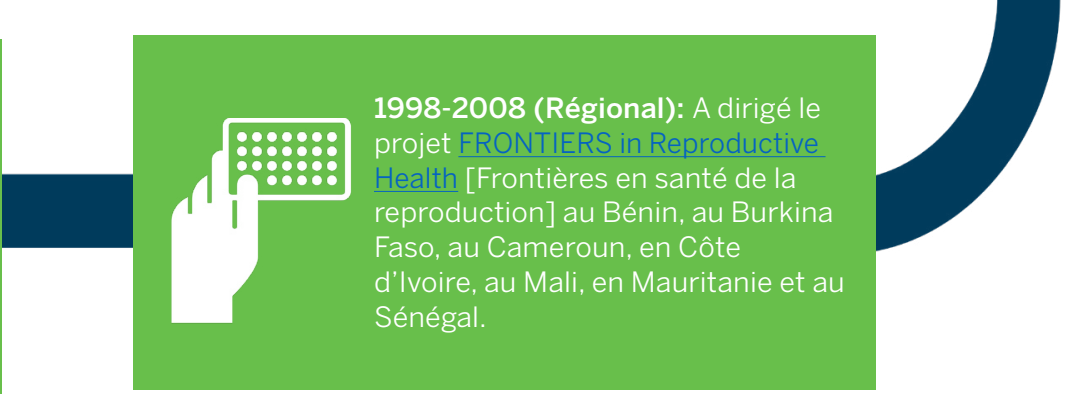

2009-2010 (Burkina Faso): A établi un partenariat avec le MS du Burkina Faso pour lui fournir un personnel détaché et une assistance technique afin de faciliter le développement d'une stratégie nationale de santé communautaire. A appuyé l'évaluation du programme du MS sur la fistule obstétricale et fourni des recommandations en vue de futurs programmes. 

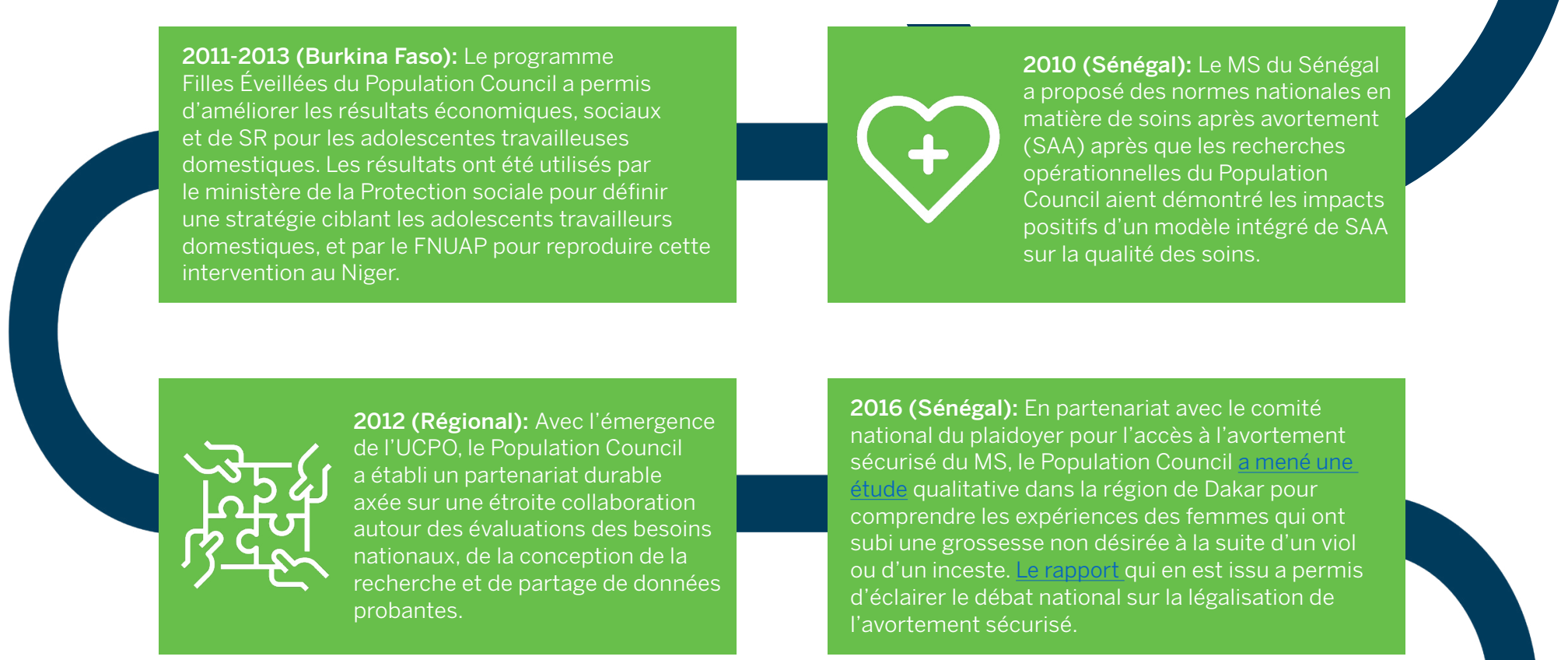

2016 (Sénégal): En partenariat avec le comité national du plaidoyer pour l'accès à l'avortement sécurisé du MS, le Population Council a mené une étude qualitative dans la région de Dakar pour comprendre les expériences des femmes qui ont subi une grossesse non désirée à la suite d'un viol ou d'un inceste. Le rapport qui en est issu a permis d'éclairer le débat national sur la légalisation de lavortement sécurisé.

2020 (Sénégal): Appuyé par une étude précédente sur l'acceptabilité de l'anneau vaginal à progestérone (AVP), le Sénégal est devenu le premier pays africain à enregistrer une méthode pour les femmes allaitantes. Le Population Council Sénégal s'est engagé dans un processus d'élaboration d'un guide national de l'auto-soin, basé sur les recommandations de l'OMS et incluant I'AVP.

\section{UTILISATION \& IMPACT DE LA RECHERCHE}

Les recherches du Population Council ont directement guidé les politiques et les programmes à travers toute l'Afrique de l'Ouest francophone et ce faisant, elles ont amélioré la planification familiale, la santé sexuelle et reproductive des adolescent-e-s et les efforts visant à prévenir la MGF/E et le mariage des enfants. L'assistance technique a aussi appuyé l'institutionnalisation infranationale, nationale et régionale de la PF/SR ; de la santé maternelle, néonatale et infantile et d'autres capacités sanitaires. Les efforts en matière de recherches et de plaidoyer ont encouragé le dialogue sur la mise en place de conditions favorables à l'accès à l'avortement sécurisé.

\section{Renforcement du partenariat régional par le biais de I'UCPO}

Depuis la création de l'UCPO en 2012, le Population Council a joué un rôle de partenaire de confiance, dans le cadre d'une collaboration étroite, pour identifier les idées de recherches en fonction des besoins des pays et en participant à la co-conception des activités de recherches. Par exemple, le Population Council, en collaboration avec I'UCPO, a appuyé une activité de I'OOAS avec I'OMS/AFRO pour documenter la délégation des tâches en matière de $P F$ au niveau communautaire dans l'ensemble des 9 pays du PO. Un rapport de 2016 faisant la synthèse de leçons apprises a été présenté lors d'une consultation régionale co-organisée par l'OMS/AFRO, le PO, l'OOAS et le Population Council, qui a appuyé le développement de plans nationaux pour le transfert de tâches relatives à la PF. 
Depuis 2014, le Population Council et I'UCPO ont aussi co-organisé un concours annuel d'excellence en production médiatiques pour les journalistes et blogueurs afin de stimuler la production d'informations et de commentaires de qualité sur la PF/la SR dans les pays du PO. Pendant cette période, le Population Council a documenté la manière dont les publications des journalistes ont favorisé un débat public sur la $\mathrm{PF} / \mathrm{SR}$. Lors de la huitième réunion annuelle du Partenariat de Ouagadougou en décembre 2019, le Population Council a partagé des informations clés tirées d'une évaluation de l'impact du travail des journalistes sur le débat public, les politiques et les changements de programmes et leur production de plus de 150 articles et reportages sur la santé reproductive dans les neuf pays du $\mathrm{PO}$.

\section{Renforcement dles opportunités des filles à travers le Sahel}

Depuis 2019, le Population Council travaille avec le bureau régional de l'Afrique de l'Ouest et du Centre de l'UNFPA en tant que partenaire stratégique au sein de l'initiative multi-pays SWEDD de la Banque mondiale. Aux côtés des gouvernements nationaux et des partenaires d'exécution, le Population Council fournit une assistance technique et un appui en termes d'apprentissage et de mise en œuvre pour améliorer l'efficacité et la durabilité des activités afin d'élargir les possibilités et limiter les risques, tels que l'abandon scolaire, le mariage des enfants et la grossesse précoce chez les filles et les jeunes femmes vivant dans la pauvreté.

Sur la base des évaluations des besoins des pays en matière de programmes SWEDD « centrés sur les filles », y compris les espaces sûrs communautaires et scolaires et les activités d'autonomisation économique, le Population Council a plaidé avec succès auprès de la Banque mondiale et des parties prenantes régionales, pour que la deuxième phase de SWEDD intègre les concepts essentiels de la programmation comme la planification segmentée pour tenir compte

de la diversité des participants, la sélection intentionnelle des communautés et la gestion adaptative. Dans le cadre de SWEDD II, le Population Council continue d'appuyer les pays pour promouvoir la programmation relative aux Espaces Sûrs fondée sur des données probantes tandis que la couverture s'élargit. Parmi les activités figurent le renforcement et la diffusion des supports pour améliorer la standardisation et le ciblage ; le suivi, l'évaluation et l'apprentissage; la gestion des mentors; la gestion de la COVID-19; et planifier une mise à échelle de qualité.

\section{POINTS FORTS DU PARTENARIAT}

\section{PARTENAIRE RÉGIONAL DE CONFIANCE}

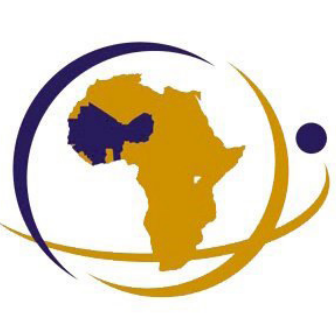

Avec l'UCPO, le Conseil a soutenu le développement de plans nationaux pour la délégation des tâches en $\mathrm{PF}$, et un concours annuel d'excellence en production médiatique pour améliorer les débats publics, les politiques et les changements de programmes.

\section{COLLABORATEUR TECHNIQUE DE VALEUR}

Avec la Banque mondiale et I'UNFPA, le Conseil a amélioré la programmation centrée sur les filles, en fournissant une assistance technique pour cette initiative multipays lancée à la demande des gouvernements nationaux du Sahel.

\section{RESSOURCE NATIONALE RÉACTIVE}

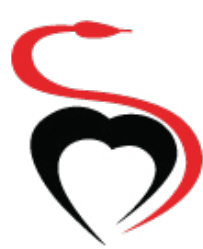

Ministère de la Santé et de l'Action Sociale
Avec le Ministère de la Santé et de l'Action Sociale du Sénégal, le Conseil a évalué le premier Plan d'Action National de Planning Familial de la région et soutient l'élaboration d'un troisième plan national de PF budgétisé. 


\section{Appui aux efforts de planification nationale en matière de PF/SR au Sénégal}

Depuis plusieurs décennies, le Population Council a travaillé en faveur de l'élargissement du choix de méthodes contraceptives dans un contexte juridique et réglementaire en évolution au Sénégal, en soutenant les efforts qui ont pour but d'améliorer l'accès aux services de qualité par le biais de guichets uniques et du transfert de tâches de même que l'avancement de la contraception d'urgence. En 2016, le Projet Evidence a évalué le Plan d'action national de Planification Familiale 2012-2015, qui était fondé sur une « Approche 3D » (Démocratisation, Démédicalisation, Décentralisation). Figurant parmi les premiers pays du PO à élaborer un PANPF, le Sénégal, par sa mise en œuvre de cette approche, a permis au Population Council de documenter les leçons apprises et de les partager avec les autres pays de la région. En étroite collaboration avec le ministère de la Santé et I'USAID/Sénégal, les résultats de cette étude ont aussi éclairé le développement du Cadre stratégique National de Planification Familiale (2016-2020) qui a suivi.

Pour faire avancer les objectifs de PF mis en exergue dans le PANPF, le Population Council a appuyé une évaluation de l'offre de méthodes contraceptives par les pharmacies privées à la demande du ministère de la Santé et d'autres partenaires. Le projet Evidence a publié deux résumés et trois rapports qui évaluent les obstacles légaux et non-légaux en matière de mise à disposition de services de PF par les pharmacies privées, parallèlement aux données qualitatives et quantitatives ayant pour but d'éclairer les recommandations politiques. Au Sénégal, ces données contribuent au développement du troisième plan national de mise en œuvre chiffré et appuie le dialogue régional sur le transfert de tâches pour la PF et l'élargissement du rôle du secteur privé.

\section{À L'AVENIR}

À l'avenir, le Population Council va poursuivre sa collaboration étroite avec les partenaires nationaux et régionaux afin d'appuyer les priorités clés à travers l'Afrique de l'Ouest francophone. À la lumière des nouveaux engagements de FP2030 et de la nouvelle stratégie du PO, le Population Council demeure un contributeur et un conseiller de confiance, qui prend part aux sessions de planification et fournit une assistance technique aux Ministères de la Santé et à ses principaux partenaires.

Conformément à ces efforts régionaux, le Population Council se réjouit de continuer à soutenir l'introduction de nouveaux outils et approches fondés sur des données probantes grâce aux échanges et à la collaboration entre pays. II reste bien placé pour répondre à des besoins en évolution constante en matière de ressources humaines et à des stratégies changeantes qui visent à une implication accrue du secteur privé. II maintiendra son attention sur l'avancement de la santé et des droits sexuels et reproductifs à travers des projets consacrés au transfert de tâches et à l'auto-soin, à l'accès à l'avortement sécurisé, à la prévention du mariage des enfants, à l'amélioration des opportunités pour les filles et à la lutte contre l'infertilité dans le cadre des efforts de soins de santé. Avec un écosystème régional de partenaires et d'investissements axés sur le renforcement des communautés résilientes, la lutte contre le changement climatique et la promotion de la paix et de la gouvernance, le Population Council demeure un partenaire engagé en Afrique de l'Ouest francophone, qui est bien positionné pour relever les nouveaux défis dans les domaines de la santé et du développement. 


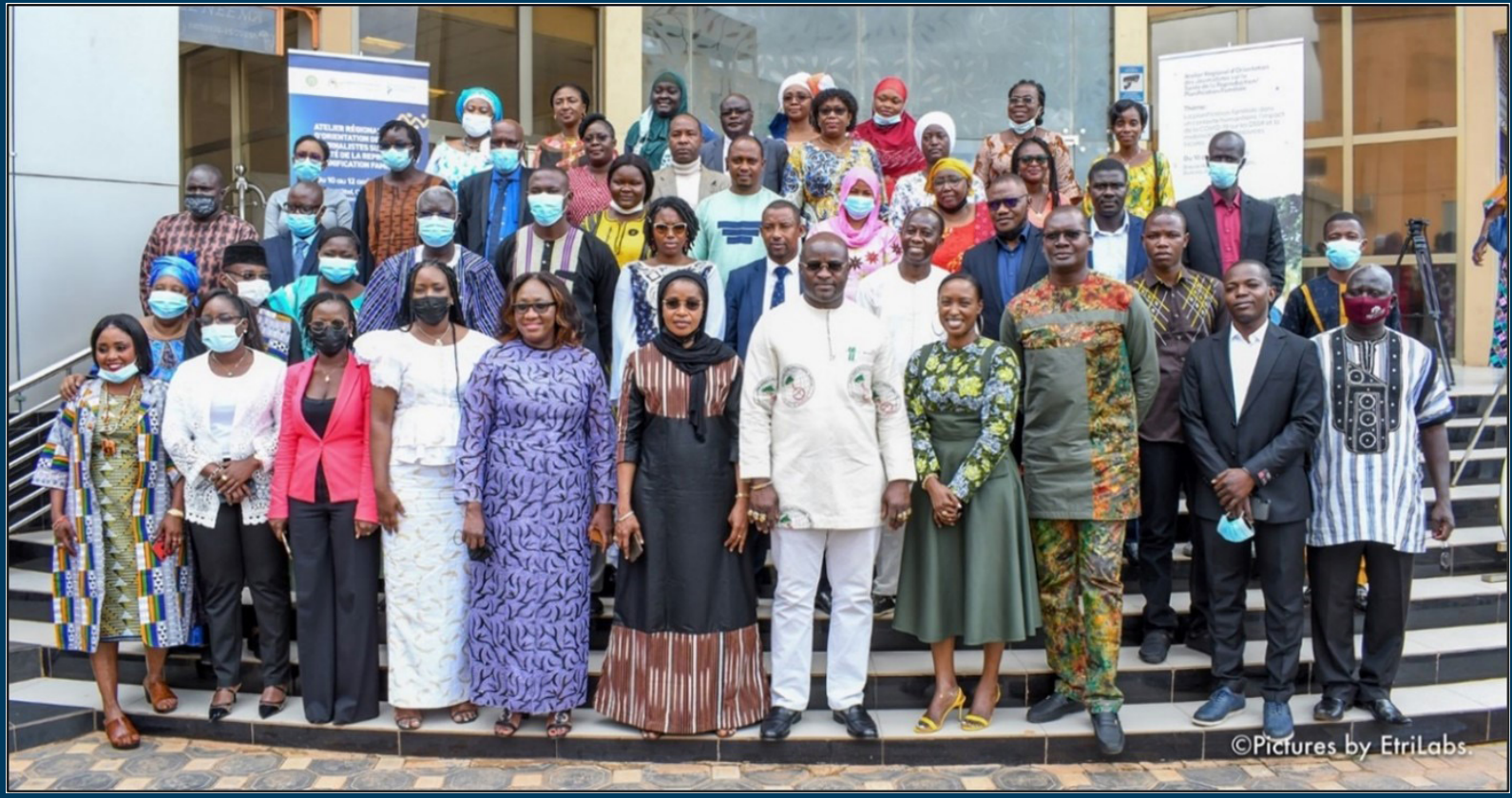

Atelier régional d'orientation des journalistes et des blogueurs sur la santé de la reproduction /planification familiale. 10 - 12 août 2021 à Ouagadougou (Burkina Faso).

\begin{tabular}{|c|c|}
\hline $\begin{array}{l}\text { Partenariat } \\
\text { avec } \\
\text { I'UCPO }\end{array}$ & $\begin{array}{l}\text { - Rapport : La délégation des tâches en matière de planification familiale au niveau } \\
\text { communautaire dans les pays du partenariat de ouagadougou : experiences et leçons } \\
\text { - } \frac{\text { apprises pour une mise en ouvre effective - Rapport de synthèse }}{\text { Report: Rapport d'évaluation du Concours d'excellence en production médiatique sur la }} \\
\text { planification familiale à l'intention des journalistes des pays du Partenariat de Ouagadougou } \\
\text { organisé par le Population Council et le Partenariat de Ouagadougou }\end{array}$ \\
\hline SWEDD & $\begin{array}{l}\text { - Guide: Le Guide des Normes Minimales pour des Espaces Sûrs: la conception, la mise en } \\
\text { - } \frac{\text { Kuvre, et le suivi, I'évaluation et l'apprentissage }}{\text { Kit pratique : Valoriser le potentiel des mentores Recrutement, formation et soutien des }} \\
\text { - } \frac{\text { mentores pour des programmes dédiés aux adolescentes }}{\text { Résumé : Prise De Mesures Pour Les Adolescentes En Periode De La Covid-19; Implications }} \\
\text { Pour La Programmation Des Espaces Sûrs Au Sahel }\end{array}$ \\
\hline $\begin{array}{l}\text { PF au } \\
\text { Sénégal }\end{array}$ & $\begin{array}{l}\text { - Résumé \& rapport : Evaluation de la mise en oeuvre et des réalisations de l'Approche 3D au } \\
\text { - } \frac{\text { Rein du Plan d'Action National de Planification Familiale (PANPF) au Sénégal }}{\text { Résumé \& rapport : Exploration du rôle potentiel des pharmacies privées dans l'offre de }} \\
\text { services de planification familiale au Sénégal (Exploration of the potential of private sector } \\
\text { pharmacies to offer family planning services in Senegal) } \\
\text { Rapport technique: Les pharmacies privées dans l'offre de services de planification familiale } \\
\text { au Sénégal: Une étude pilote }\end{array}$ \\
\hline
\end{tabular}

Le Population Concil travaille en collaboration avec des responsables de programme, des décideurs, des chercheurs et des partenaires bailleurs de fonds pour faire avancer les solutions fondées sur des données probantes, face aux problèmes de santé et de développement. Ses résumés consacrés à l'utilisation et à l'impact des recherches exposent les points clés de notre travail en cours, le but étant de faire en sorte que les données probantes soient traduites en actions significatives afin d'améliorer les conditions de vie au niveau communautaire, national, régional et mondial.

Citation recommandée : Population Council. «Traduire les données probantes en impact: appuyer la santé en Afrique de l'Ouest francophone et dans le Sahel » Résumé sur l'utilisation et l'impact de la recherche. Washington, D.C. : Population Council, 2022.

Crédit photo sur la page de garde: (c Dominic Chavez/ Le Mécanisme de Financement Mondial (GFF) 